\title{
Electrophysiological correlates of tactile remapping
}

\author{
Salvador Soto-Faraco ${ }^{\mathrm{a}, \mathrm{b}}$ and Elena Azañón ${ }^{\mathrm{a}, \mathrm{c}}$ \\ aDepartament de Tecnologies de la Informació i les Comunicacions, Universitat Pompeu Fabra, \\ Barcelona 08018, Spain \\ ${ }^{\mathrm{b}}$ ICREA, Institució Catalana de Recerca i Estudis Avançats, Barcelona 08010, Spain \\ 'Institute Cognitive Neuroscience, University College London, WC1N 3AR London \\ ${ }^{*}$ Corresponding author: \\ Salvador Soto-Faraco, \\ Address \\ Dep.de Tecnologies de la Informació i les Comunicacions, Roc \\ Boronat 138, Barcelona 08018, Spain \\ E-mail address: \\ salvador.soto@icrea.cat \\ Phone: \\ +34 93.542.25.34 \\ Fax: \\ +34 93.542.25.17 \\ Second author: \\ Elena Azañón \\ E-mail address: \\ eazanyon@gmail.com
}




\section{Abstract}

Orienting our gaze or attention to the location of tactile events in the skin feels natural and effortless. However, this process requires combining somatosensory and proprioceptive information in a non-trivial, time consuming, fashion. Here we address the time course of tactile remapping, from somatotopically-based representations, to a spatiotopic reference frame. We compared electrical responses at the scalp evoked by touch at one finger as participants held their arms straight or else, crossed about the body midline. This postural manipulation creates a conflict between frames of reference, which can be used to reveal the consequences of spatial remapping. Behavioural performance was gauged online for crossed and uncrossed postures using bimanual temporal order judgment (TOJ) trials occurring occasionally during the recording session. The first electrophysiological signs of tactile remapping were observed around $70 \mathrm{~ms}$ after the tactile event, overlapping in time with the somatosensory component N80. This electrophysiological effect was strongly lateralized to the left scalp and independent of the hand being stimulated. What is more, participants that manifested a stronger behavioral conflict between frames of reference in the TOJ task displayed a larger electrophysiological effect. Based on these findings, and the known properties of the somatosensory network, we argue that remapping of tactile space depends on fast feedback projections from association areas of the parietal cortex, and encompasses a left-lateralized fronto-parietal network supporting the selection of guided actions.

\section{Keywords}

Tactile processing; Somatosensory evoked potentials; Spatial reference frames; Hemispheric lateralization; Temporal order judgments. 


\section{Introduction}

Representing the spatial origin of sensory stimulation is of the essence in many of the motor and cognitive behaviours displayed by most animals, including humans. However, the coordination between the various spatial reference frames in which information is encoded represents a fundamental challenge. For example, visual stimuli are initially represented in retinotopic coordinates, but oculomotor behaviour unfolds in a head/trunk-centered coordinate system (Duhamel et al., 1997; Avillac et al., 2005; Schlack et al., 2005). Similarly, in touch, sensations on the skin are initially projected to somatotopically-organized maps in somatosensory cortex, and then quickly referred to an external frame of reference that represents information relative to some body part, thus providing an adequate read-out for effector systems (Pouget and Sejnowski, 1997; Cohen and Andersen, 2004). Here we address the time course of this tactile remapping process, and in particular, we track down its earlier stages. Our aim is to infer some of the functional properties of the brain circuit that supports this relatively unknown aspect of human spatial processing.

Evidence for the dominance of an external reference frame in the execution of tactile tasks comes from deficits in brain damaged patients (e.g., Moscovitch and Behrmann, 1994; Aglioti et al., 1999) and posture-related studies in healthy humans, often with crossed-hands (Soto-Faraco et al., 2004; Spence et al., 2004). Crossing the hands over about the body midline induces a misalignment between the anatomical location in the body and the external origin of tactile events, thus offering a chance to dissociate the dominance of one or the other type of representation. Several prior studies show that cross-modal interactions (Spence et al., 2004) and attention orienting (Kennett et al., 2001) tested with crossed hands often abide to an external frame of reference. Thus, in cross-modal cueing, a touch at one hand facilitates detection of upcoming visual targets (presented 200-350 ms later) near that hand, regardless of where the hand is placed in space (Kennett et al., 2001; Kennett et al., 2002). Interestingly, however, Azañón and Soto-Faraco (2008a) found that at shorter cue-target intervals (30-60 $\mathrm{ms}$ ), this spatial validity effect depends instead on the anatomical side of the stimulated hand, reflecting the initial somatotopic reference frame for touch (see also Azañón and Soto-Faraco, 2008b; Azañón et al., 2010a). According to these results, tactile representations undergo a transition between an anatomical and an externally-based reference frame (between 60 and 
$360 \mathrm{~ms}$ ). Similar conclusions regarding the timing of remapping have been inferred from studying saccades to tactile targets at the hands. In particular, when the arms are crossed, quick saccades to tactile events are often curved ( 190 ms; Groh and Sparks, 1996; Overvliet et al., 2011). In line with this idea, bimanual tactile temporal order judgments (TOJ) at short inter-stimulus intervals are often reversed in space when the hands are crossed (Yamamoto and Kitazawa, 2001; Kóbor et al., 2006). In particular, the perceived temporal order of two successive tactile evens is often reversed at intervals shorter than 300 ms (e.g., Yamamoto and Kitazawa, 2001; Shore et al., 2002), providing an estimate for how long it takes to refer a tactile event in external space (Kitazawa, 2002).

It is important to understand the sequence of neural events leading from anatomicallyto externally-based representations, and to help infer its underlying brain network. Although some studies report modulation of somatosensory-evoked neural activity by online finger movement (Huttunen et al., 1996), evidence for the influence of an external reference frame on neural activity is mostly confined to spatial attention paradigms. In particular, spatial attention effects on the somatosensory evoked potentials (SEPs), typically expressed in mid-latency components (from N80; Eimer and Forster, 2003), are enhanced by increasing hand separation (Eimer et al., 2004; Gillmeister et al., 2010) and abolished by hand crossing (Eimer et al., 2001; Eimer et al., 2003). These results suggest that external space is already taken into account when spatial attention is deployed, though the influence of an anatomical reference frame remains evident at latencies up until 100-140 ms (Heed and Röder, 2010). Two recent fMRI studies have addressed tactile temporal order tasks across different arm postures (Takahashi et al., 2012; Wada et al., 2012). Despite the many differences between these studies in methodology and focus, their results reveal surprising coincidences. First, both studies highlighted the relevance of posterior parietal areas for the spatial updating of tactile representations across postures (in line with Azañón et al., 2010b; Bolognini and Maravita, 2007). In addition, Takahasi et al. reported the activation of frontal areas, thus suggesting the importance of a coordinated fronto-parietal network (see also Lloyd et al., 2003). Second, in both studies, the comparison between crossed and uncrossed postures resulted in stronger/more extended BOLD responses in the left hemisphere. 
Here, and in contrast to these previous fMRI studies, we used SEPs to concentrate on the temporal course of remapping. In addition, contrary to most electrophysiological research to date, we addressed tactile remapping outside the context of spatial attention. That is, we did not study the effects of attended vs. unattended events. We instead focused on the moment at which the spatial representation of the tactile event starts to be encoded in external coordinates, rather than the timing at which the spatial allocation of attention is expressed in such terms. To this aim, we compared SEPs to single, predictable, finger stimulation with hands uncrossed (i.e., when anatomical and external frames of reference are better aligned) and with hands crossed (i.e., when anatomical and external frames of reference are placed in conflict). Under these conditions of focused, constant, spatial attention we aimed at inferring the functional significance of SEP modulations by way of their timing, scalp distribution, and correlation with behavioural measures of remapping.

In the study, a single tactile tap was presented to the left hand in one group of subjects and to the right hand in a second group of people. Interleaved within the stream of frequent single taps, we included infrequent trials requiring a bimanual temporal order judgment task, so we could index the cost of remapping between reference frames behaviorally. As mentioned above, we ran two separate sets of participants, each receiving the stimulus stream at a different hand, to pinpoint any possible lateralization effects regarding tactile remapping (Lloyd et al., 2003; Takahashi et al., 2012; Wada et al., 2012).

\section{Materials and Methods}

\subsection{Participants}

Forty-seven healthy adult volunteers participated in the study, of whom twenty-five were tested with the left hand (LH experiment) and twenty-two with the right hand (RH experiment). Seven volunteers from the $\mathrm{LH}$ experiment and eight from the $\mathrm{RH}$ experiment were excluded from the final analyses according to the following criteria: (1) unacceptable number of EEG artefacts (affecting $>60 \%$ of the SEP data; $\mathrm{LH}: 3, \mathrm{RH}: 4$ ) and (2) poor performance on the behavioural task (based on outlier rejection analysis, Rosner, 1975, on the following parameters: $\sigma, P_{\max }$ and $P_{\min }, r^{2}$; see Equation 1; LH:4, RH:4). Thus, the LH experiment was based on 18 participants (12 
female) with mean age of 25 years (20-36 years), right handed and normal tactile sensitivity.

The RH experiment included 14 (10 female) participants with 24 years average age (also right handed). All were naïve as to the purpose of the experiment and gave written informed consent prior to the study, which was approved by the local ethics committee.

\subsection{Materials and procedure}

Participants sat at a comfortable chair, wearing the EEG recording cap and a solenoid tapper (rounded tip $\varnothing 0.2 \mathrm{~mm}$; M\&E Solve, Kent, UK) strapped to the middle finger of each hand (dorsal aspect of the middle phalange) at a constant, comfortable, pressure. They placed both hands, crossed or uncrossed depending on the block, on a flat wooden plate placed on their lap, with their middle fingers resting each on a response button, $30 \mathrm{~cm}$ apart. An orange LED, in front of the participant, served as fixation. Otherwise, the room was kept dark and the hands were visible only under the dim illumination of the fixation light.

Each participant was presented with 2,240 trials. Amongst these 2,000 were single tactile events ( 9 ms mechanical suprathreshold tap driven by a 12-v square wave) at the middle finger of a single hand (i.e. the left or right hand for the $\mathrm{LH}$ and $\mathrm{RH}$ experiments respectively). Participants wore disposable foam earplugs and earmuffs to prevent hearing the stimulator noise. The single taps were presented in a continuous stream at a rate of $\sim 1.33 \mathrm{~Hz}$ (Stimulus Onset Asynchrony -SOA- jittered between 600-900 ms). In order to maintain the participants' alert state and to gauge behavioural performance during the recording session, we included a bimanual TOJ task which allowed us to index the cost of conflict between spatial reference frames (Yamamoto and Kitazawa, 2001; Shore et al., 2002; Röder et al., 2004; Schicke and Röder, 2006; Azañón and Soto-Faraco, 2007; Collignon et al., 2009; Heed et al., 2011). In TOJ tasks, the perceived temporal order of two successive tactile events, one at each hand, is often erroneously reversed at inter-stimulus intervals shorter than $300 \mathrm{~ms}$ (e.g., Yamamoto and Kitazawa, 2001; Wada et al., 2004; Kobor et al., 2006). This has been proposed to reflect the time it takes to refer a tactile event in external space, with the second touch interfering with the remapping process of the first touch (see also Kitazawa, 2002). Thus, randomly intermingled within the sequence of single taps at one particular hand (left or right, depending on group), an extra $\sim 10 \%$ of trials (240 total) consisted of a double tap, one at each hand. The SOA and hand 
order between taps in double-tap trials was sampled equiprobably following a constant stimulus distribution $( \pm 10, \pm 30, \pm 60, \pm 180, \pm 360$ and $\pm 900 \mathrm{~ms}$; where negative values indicate left hand first stimulation). After double tap trials, the sequence of single taps at the designated hand stopped and participants were prompted to press a button with the finger that had received the last stimulus of the sequence to resume the single tap stimulations. These double-tap trials were not included in the SEPs analyses, and were only used to collect online psychophysical data on TOJ performance individually. The fixation LED was illuminated throughout the experiment, except when a manual response was required from the participant. That served as a prompt for response.

The experimental session was organized in 10 blocks of 224 trials (200 single taps with no response required, 24 double taps for the TOJ task) with short resting breaks in between. Posture (arms straight or crossed about the midline, left over right) was alternated between blocks following an $A B B A B A A B A B$ sequence (starting posture was counterbalanced across participants).

\subsection{Recording parameters}

The EEG was continuously recorded from 30 tin electrodes (10-20 system, 27 scalp locations Fp1/2, F3/4, F7/8, FC3/4, C3/4, Cp3/4, FT7/8, T3/4, T5/6, P3/4, O1/2, Fz, FCz, Cz, Cpz, Pz) mounted in an elastic cap (Brain Vision Recorder 1.05; Brain Products). Two bipolar electrodes were placed beneath and at the outer canthus of the left eye (EOGH and EOGV) to register eye movements, and one electrode was placed at the tip of the nose, to which all the remaining electrodes were referenced. Electrode impedances were kept below $2 \mathrm{k} \Omega$ for the reference and ground electrodes, and lowered to $<3 \mathrm{k} \Omega$ for all other electrodes prior to recording. The recordings were made at a sampling rate of $512 \mathrm{~Hz}$. Offline pre-processing imposed an additional $0.07-40 \mathrm{~Hz}$ band pass filter on the EEG, and the removal of a $200 \mathrm{~ms}$ window centred around ocular movements, blinks and other artefacts on individual basis (voltage deviations of $\pm 100 \mu \mathrm{V}$ in any electrode, and/or beyond $\pm 70 \mu \mathrm{V}$ in EOGh, EOGv, Fp1 or Fp2). Trials surviving the rejection procedure were segmented out, time locked with stimulus presentation (-100 ms to $500 \mathrm{~ms}$ ), baseline corrected, and averaged for each individual as a function of posture (crossed or uncrossed). 


\section{Data analysis}

\subsection{Behavioural analyses.}

We calculated the average proportion of right-first responses (i.e., left second responses) for each participant, arm posture (crossed, uncrossed) and SOA (-900 to $+900 \mathrm{~ms})$ for each experiment $(\mathrm{LH}, \mathrm{RH})$. The individual data were then fitted to a cumulative Gaussian function limited by an upper and lower asymptote (so, with the parameters mean, standard deviation and lower and upper asymptotes, see Equation 1). The standard deviation of the Gaussian fit indexes the precision in the temporal order task (i.e., just noticeable difference; JND in time between two stimuli at $84 \%$ ), and the mean is a measure of bias that corresponds to the point of subjective simultaneity between the two stimuli (PSS, hereafter). A repeated-analyses ANOVA on these two parameters with hand group as a between-subject factor was conducted.

\section{EQUATION 1 ABOUT HERE}

\subsection{Electrophysiological analyses.}

The first analytical step was to reveal the timing at which the electrophysiological recording began to differ between postures. Because this question is in principle independent of any particular component in the event related potential (see Luck, 2005, for an extension of this approach; see also Thorpe et al., 1996; Dambacher et al., 2009, for examples), we calculated difference weaves between the SEPs from crossed and uncrossed postures for each electrode and ran point by point comparisons (time by electrode analyses). To minimise the probability of Type I error, we considered only differences that survived both of two criteria; a significance threshold $P<.05$ over 10 subsequent $\mathrm{ms}$, and a M/EEG-cluster-based nonparametric permutation test (Maris and Oostenveld, 2007) ${ }^{1}$. In a second step, to pinpoint any possible findings to known somatosensory components, we also organized the data within pre-defined 
temporal windows around the classical components of the somatosensory ERP: P35 (20-40 $\mathrm{ms}), \mathrm{P} 50$ (40-60 ms), N80 (70-90 ms), P100 (90-110), N140 (120-160 ms), first part of the Late Positive Deflection (LPDa, 200-300 ms) and second part of the Late Positive Deflection (LPDb, $300-400 \mathrm{~ms})$. Note that this analysis was not based on the earlier point-by-point analysis, and it therefore provides independent evidence. For each of these components, we submitted the voltage data to an ANOVA that included Posture (crossed, uncrossed), Hemisphere (left/right), position in the coronal axis (inferior: F7/8, FT7/8, T3/4, T5/6; superior: F3/4, FC3/4, C3/4, $\mathrm{CP} 3 / 4$ ) and position in the sagittal axis (from anterior to posterior: F7/8 \& F3/4; FT7/8 \& FC3/4; $\mathrm{T} 3 / 4$ \& $\mathrm{C} 3 / 4$; and $\mathrm{T} 5 / 6$ \& $\mathrm{CP} 3 / 4)$ as within-subject factors. Hand group was included as a between-subject factor. The dependent measure was mean amplitude at each electrode site. The Greenhouse-Geisser correction was applied to adjust degrees of freedom and $P$-values for violations of the sphericity assumption. In addition, further partial ANOVAs and post hoc paired t-tests were performed after interactions. Effects for the factors hemisphere, sagittal and coronal positions are only reported when they interacted with the experimental manipulation (i.e. Posture). We also included analyses at the individual group level ( $\mathrm{LH}$ and $\mathrm{RH}$ separately), to strengthen or diminish any possible overlapping effects across experiments, which are in principle independent. Finally, we sought for correlations between the electrophysiological effects that had turned out significant and the individual performance in the TOJ task.

\section{Results}

\subsection{Behavioural results}

The data was well conformed to the cumulative Gaussian fit for both experiments (all average $r^{2}>.91 ;$ all $S D<.09$ ). The TOJ results estimated from the Gaussian fits (see above) confirmed a crossed hands deficit, revealing a two-fold increase in JND (i.e., decrease in precision) when participants performed the task with hands crossed compared to uncrossed, indexing the higher probability of reversals in the former posture (103 vs $\left.47 \mathrm{~ms} ;\left[F_{(1,30)}=19.91 ; P<.001\right]\right)$. This posture effect on TOJ precision was independent of the stimulated hand, as shown by the lack of group by posture interaction $[F<.1 ; P>.75]$, and it was further confirmed at the group level 
$\left(\mathrm{LH} ;\left[t_{(17)}=3.82, P=.001\right] ; \mathrm{RH} ;\left[t_{(13)}=2.59, P=.022\right]\right.$; see Figure 1$)$. The lower and upper asymptotes did not differ statistically between crossed and uncrossed conditions, indicating that simple confusions between left and right hand at the time of response cannot explain this difference in performance across postures (all PS>.15). Regarding the PSS, there was a main effect of group $\left[F_{(1,30)}=34.68 ; P<.001\right]$, revealing a shift toward perceiving taps on the frequently stimulated hand as occurring earlier than taps at the alternate hand (the right hand in the $\mathrm{RH}$ group, PSS $=+85 \mathrm{~ms}$, and vice versa for the LH group, PSS $=-100 \mathrm{~ms}$ ). This PSS effect could reflect the Bayesian prior introduced by the higher frequency of occurrence of the stimulated hand events (see Miyazaki et al., 2006; Yamamoto et al., 2012) or else, it may relate to the well known phenomenon of prior entry, whereby attended stimuli tend to be perceived sooner than unattended ones (Yates and Nicholls, 2009, 2011). This PSS effect was stronger in the crossed posture, as indicated by a posture $\mathrm{x}$ group interaction $\left[F_{(1,30)}=7.02 ; P=.013\right]$, but at the group level, the effect of posture was only marginally significant $\left(\mathrm{LH} ;\left[t_{(17)}=2.05, P=.056\right] ; R H ;\left[t_{(13)}=-\right.\right.$ $1.80, P=.096])$.

\section{FIGURE 1 ABOUT HERE}

\subsection{Electrophysiological results}

The main cortical SEP components typical of a somatosensory evoked potential could be visually identified in the signal (Allison et al., 1989a; Allison et al., 1989b), both in the LH and $\mathrm{RH}$ experiments, except for the very early $\mathrm{N} 20$, whose absence was due to limited resolution and the electrical artefact related to the voltage injection driving the stimulators (lasting for about $16 \mathrm{~ms}$ ). This qualitative profile is illustrated in Fig. 2a for right and left hand stimuli for two electrodes. An early deflection around $30-35 \mathrm{~ms}$, probably corresponding to the P35 component (see electrode C4, in Fig. 2a, top panel) was seen, followed by a distinct (but overlapping in some electrodes) contralateral positive deflection corresponding to P50. The N80 component was seen most clearly over contralateral electrodes (e.g., C4 for LH stimulus, and FT7 for RH stimulus, in Fig 2a), though also ipsilaterally (e.g., T5, CP3 for LH experiment; C4, in Fig 2a, for RH stimulus). The first, clearly bilateral component was P100 over frontal electrodes 
(F3/4, FC3/4, C3/4), followed also bilaterally by the N140 over the temporo-parietal scalp. After the $\mathrm{N} 140$, a large positive deflection followed throughout the scalp, peaking at different times between 220 and $350 \mathrm{~ms}$ at different electrodes. Activity returned to baseline for all electrodes by the end of the segment ${ }^{2}$.

\section{FIGURE 2. ABOUT HERE}

\subsubsection{Time by electrode analysis}

Figure 3 provides a visual summary of the initial point-by-point t-test comparisons for crossed vs. uncrossed ERPs, for each group ( $\mathrm{LH}$ and $\mathrm{RH})$. This difference wave analysis revealed an initial reliable difference around the 70-90 ms window, concentrated on a fronto-temporal group of adjacent electrodes over the left scalp. Remarkably, these differences appear to be similar amongst groups both in polarity (negative shift for crossed hands; see Fig. 2b) and spatial distribution (electrodes F7, FT7 and T3, for the LH stimulation and FT7 and T3 for the RH). No other reliable effects were seen in the difference wave until the $170-280 \mathrm{~ms}$ time range (also negative shifts), which were visible over a cluster of temporal and posterior electrodes again at the left scalp (T3, T5, CP3, P3 and O1 for the LH group and F3, FC3, C3, CP3 for the RH group and extending more centrally and anteriorly 50 ms later $-\mathrm{F} 7, \mathrm{FT} 7, \mathrm{FCz}, \mathrm{Cz}, \mathrm{CPz}$ for the $\mathrm{RH}$ group). This negative shift continued later on within the $300-350$ range (again, predominantly over left and central electrodes, but extending later on over right scalp).

Visual inspection of Figures $2 \mathrm{~b}$ and 3 reveals a striking similarity between the groups regarding the timing and the scalp distribution of the differences in somatosensory evoked potentials as a function of posture, despite these data correspond to different participants stimulated in different hands. In particular, three features emerging from of this pattern of results are worth noting; (1) the earliest reliable difference in somatosensory evoked potentials related to postural manipulation starts as early as $70 \mathrm{~ms}$ after stimulation at the skin; (2) the effect of posture is characterized as a negative shift for crossed hands; (3) these posturerelated effect on the SEP express on the left scalp. 


\subsubsection{Analysis by temporal window}

No effect of posture was found in the early time windows P35 and P50 (all PS $>0.12$ ). Thus, we can assume that the somatosensory evoked potentials are equivalent regardless of posture up to $60 \mathrm{~ms}$. The first relevant significant difference was detected in the N80 window, with an interaction Posture $x$ Hemisphere $\left[F_{(1,30)}=6.27, P=.018\right]$, which was independent of the experimental group and therefore of the hand being stimulated $\left[F_{(1,30)}=2.1, P=.16\right]$. Post-doc paired t-tests confirmed a significant negative shift in the SEP for crossed hands posture compared to uncrossed in the left hemisphere $\left[t_{(31)}=2.01 ; P=.045\right]$ but not the right one $\left[t_{(31)}=.59\right.$; $P=.56]$. Moreover, a Posture $\times$ Hemisphere $\times$ Coronal position interaction resulted marginally significant $\left[F_{(1,30)}=3.24, P=.08\right]$, illustrating that this negative shift in the left hemisphere (Posture $x$ Hemisphere interaction) was stronger in inferior electrodes (left inferior: $\left[t_{(31)}=3.0 ; P=.005\right]$; left superior: $\left[t_{(31)}=1.4 ; P=.17\right]$; right hemisphere, all $\left.P S>.57\right)$. Along these lines, only adjacent electrodes of the left inferior scalp rendered significant differences between postures at the level of individual electrodes, precisely at the same cluster of left scalp electrodes highlighted in the point-by-point analysis within this latency (F7, FT7 and T3; all $t_{(31)}>2.5$, all $P_{\mathrm{S}}<.01$; Fig. $2 \mathrm{~b}$ shows the electrodes that rendered significant differences for each experimental group).

Even though the variable stimulated hand did not participate in any interaction in the ANOVA (all Ps>0.16), we performed separate analyses at the group level. This was done to reassure that the pattern observed in this fronto-temporal cluster of left electrodes for the whole sample of participants was reliable in each group individually ( $\mathrm{LH}$ experiment and $\mathrm{RH}$ experiment). This was indeed confirmed for the LH group (significant interaction Posture $\mathrm{x}$ Hemisphere $\times$ Coronal position; $\left[F_{(1,17)}=9.95, P=.006\right]$; see Fig 2B for significant electrodes); and for the $\mathrm{RH}$ group where the interaction Posture $\mathrm{x}$ Hemisphere $\left[F_{(1,13)}=5.21, P=.04\right]$ indicated left lateralization without variation in coronal position (see Fig 2B for significant electrodes). Thus, these results confirm a strongly lateralized pattern regardless of the stimulated hand and group of participants. 
Regarding the P100 and N140 windows no significant effects or interactions were found

involving posture (all $P \mathrm{~S}>$.12), except for a four way interaction (Posture $\mathrm{x}$ Coronal $\mathrm{x}$ Sagittal $\mathrm{x}$ Group, $\left.\left[F_{(3,90)}=4.49, P=.005\right]\right)$ in the $\mathrm{N} 140$ window. Breaking down this interaction with smaller ANOVAs and with simple t-tests (crossed-uncrossed at every condition combination) did not render any indication of further significant effects of posture. From Fig $2 \mathrm{~b}$. it would seem that there is a trend for the N140 window to be more negative ipsilateral to stimulation (thus, depending on group), but the lack of statistical confirmation we cannot interpret this interaction any further.

Differences as a function of posture were seen again in the late positive deflection window (LPDa, 200-300 ms), with a main effect of Posture $\left[F_{(1,30)}=5.17, P=.03\right]$ as well as a Posture $\mathrm{x}$ Hemisphere interaction $\left[F_{(1,30)}=5.59, P=.025\right]$, revealing again the left lateralized pattern of differences $\left(\left[t_{(31)}=3.2 ; P=.003\right]\right.$; right hemisphere: $\left.\left[t_{(31)}=1.3 ; P=.21\right]\right)$, Indeed, at the level of individual electrodes, differences as a function of posture were seen exclusively at the left (F3, FC3, F7, FT7, T3, T5, C3, CP3 and P3; all Fs>2.1; all $\left.P_{\mathrm{s}}<.05\right)$ and central scalp electrodes $(\mathrm{CPz}$ and $\mathrm{Pz}$; both $P \mathrm{~s}<.03)$. No interactions involving stimulated hand and posture were significant in this latency window, except for Posture $x$ Coronal $x$ Sagittal position $x$ hand group $\left[F_{(2.2,65.8)}=3.09, P=.048\right]$. This four way interaction in the late time window may reflect that the tendency for the effects to become bilateral with time (at longer time windows) started earlier in the $\mathrm{RH}$ experiment than in the LH experiment. This can be seen more graphically in Fig. 3, and in partial analyses per group: In the LH group the interaction Posture $x$ Hemisphere X Sagittal position was significant $\left[F_{(2.6,45.8)}=5.15, P=.005\right]$, showing a negative shift under crossed hands posture for the two posterior clusters of electrodes of the left scalp (superior: $\left[t_{(17)}=2.41, P<.03\right]$, inferior: $\left[t_{(17)}=2.21, P=.04\right]$, all others $\left.P S>.12\right)$; In the RH group the ANOVA returned only a nearly significant main effect of posture $\left[F_{(1,13)}=4.18, P=.06\right]$, and no interaction of posture with any other factor (all Ps>0.12).

Finally, for the LPDb window we found a pattern of main effects and interactions similar to that observed in LPDa, involving a main effect of Posture $\left[F_{(1,30)}=9.61, P=.004\right]$ and an interaction Posture $\times$ Hemisphere $\left[F_{(1,30)}=6.62, P=.015\right]$. Nonetheless, the effects of posture, although stronger in the left hemisphere $\left[t_{(31)}=3.5 ; P=.001\right]$, were also significant in the right hemisphere $\left[t_{(31)}=2.6 ; P=.015\right]$. In addition a Posture $\times$ Coronal position $\left[F_{(1,30)}=8.41, P=.007\right]$, 
illustrates the more birateral nature of the effects at this later time window. This tendency for the differences to become bilateral can be seen in the time by electrode comparisons (Fig 3), as well as at the at the electrode level comparisons. In this time window the significant differences remained within a large cluster of left scalp electrodes (i.e. F3, FC3, F7, FT7, T3, T5, C3, CP3, plus 01 , all $t \mathrm{~s}_{(31)}>2.5$, all $\left.P \mathrm{~s}<.02\right)$ and extended toward midline and right scalp electrodes ( $\mathrm{Fz}$, FCz, Cz, Cpz, Pz, all ts (31) $>2$., all Ps<.019; F4, FC4, C4, CP4, P4, T4, T6 and O2, all ts (13) $>2.1$, $P s \leq .044)$. The ANOVA returned no interactions involving posture and group. However, we tried to confirm the pattern found in the pooled analysis splitting the analyses by group. In the LH group we found a significant main effect of Posture $\left[F_{(1,17)}=4.26, P=.05\right]$, as well as a three-way interaction Posture x Coronal $x$ Sagittal interaction $\left[F_{(2,35.6)}=3.81, P=.03\right]$. This three way interaction was due to a significant Posture $\mathrm{x}$ Sagittal position interaction at inferior electrodes $\left[F_{(1.9,33.2)}=4.49, P<.02\right]$ but not at superior electrodes $(P>.61)$. For the RH group, the ANOVA returned a main effect of Posture $\left[F_{(1,13)}=6.6, P=.023\right]$, a marginal Posture x Hemisphere interaction $\left[F_{(1,13)}=3.9 ; \quad P=.07\right]$, and a significant interaction Posture $\mathrm{x}$ Coronal position $\left[F_{(1,13)}=8.19, P=.013\right]$. This indicated that the negative shift in crossed hands posture was dominant in the superior (more central) electrodes $\left[t_{(13)}=2.7 ; P=.017\right]$, though it was also significant in inferior electrodes $\left[t_{(13)}=2.2 ; P=.045\right]$.

Summarizing, the results show that the early SEP modulation by posture was more prominent over the left scalp, the hemisphere contralateral to the stimulated hand for the right hand group, but ipsilateral for the left hand group. These left-lateralized differences regardless of hand stimulated are therefore suggestive of a functional asymmetry ${ }^{3}$. Later on, differences tended to become more bilateral in both groups of participants.

\subsection{Correlation between Behaviour and SEPs}

Since the electrophysiological results have revealed that the earliest differences in somatosensory ERPs related to body posture are seen reliably around the N80 window (70-90 $\mathrm{ms}$ ), we ran a correlation analyses between the behavioural marker of remapping (indexed by the difference in precision between crossed and uncrossed hands in the TOJ task) and the size of the negative shift in SEPs within the N80 window. The magnitude of the N80 shift correlated significantly with behavioural performance in four electrodes, all of them in the left hemisphere 
(T3, T5, P3, 01; all $P<.05$, uncorrected). In particular, the larger the negative shift in SEPs, the larger the crossed-hands deficit in the TOJ task (i.e. larger JND differences; worst performance). None of these correlations survives a Bonferroni correction for multiple comparisons across the whole set of electrodes $(P<.002)$, but it is remarkable that, consistent with the electrophysiological analyses above, these significant correlations were observed exclusively over left scalp electrodes. When breaking down for group, we observed a strong correlation in the LH, which encompassed a similar cluster of left-hemisphere electrodes as in the pooled data, (T3, CP3, T5, P3, O1; threshold $P<.0028$ with Bonferroni correction). The results of this group are shown in Fig. 4. Again, this set of electrodes encompassed the relevant cluster in the ANOVA, plus neighbouring channels extending posteriorly. In particular, the left scalp electrode T3, displaying a significant negative shift in the N80 window, showed the strongest correlation of all the electrodes in both hemispheres $\left[r^{2}=.57, P=.0002\right]$. The RH group did not show any significant correlations at the N80 window.

We ran the same correlation analyses for the other two time windows that rendered significant posture effects in the ANOVAs. The LPDa window did not reveal any correlation. For the LPDb (300-400 ms) window, a cluster of nearby centro-parietal electrodes showed a significant correlation with the crossed hands effect (T3, P3, Cp3, C3, Pz, Cpz, Cz, FCz, FC4, $\mathrm{C} 4, \mathrm{Cp} 4, \mathrm{~T} 4, \mathrm{P} 4 ; \mathrm{P}<.05$, uncorrected) in the same direction as with the N80 window. These correlations did not survive Bonferroni correction, but again, it must be noted that the scalp distribution of the best correlated electrodes resembles the bilateral distribution of effects at this late time window in the ANOVA (see also, Fig 3). Similar patterns were seen for each group of participants independently (for the LH group Cp3, P3, Cpz, and Pz,; for the RH group F3, F4, FC3, FC4, FCz, FT8 and Fz, both at $P<.05$, uncorrected).

\section{FIGURE 4 ABOUT HERE}

\section{Discussion}

The present study aimed at revealing the neural signature of the initial steps of tactile remapping. To this goal, we compared SEPs to one finger as the participant adopted two 
different arm postures, hands uncrossed (where anatomical and external reference frames are in good alignment) and hands crossed (where anatomical and external reference frames are in spatial conflict). Because, several tactile tasks using crossed hands have revealed strong interference between spatial reference frames in the course of remapping (Nicoletti et al., 1984; Yamamoto and Kitazawa, 2001; Shore et al., 2002) we expected to register electrophysiological differences between these two conditions.

First, we found that the initial signs of tactile remapping taking place as a function of body posture arise at around $70 \mathrm{~ms}$ latency $(80 \pm 10 \mathrm{~ms}$ window) consisting of a negative deflection for the crossed-hand posture. This effect overlaps in time with the somatosensory component N80. Second, this effect was clearly lateralized to the left scalp, at fronto-temporal electrodes at early stages extending to left temporo-parietal electrodes at about $170 \mathrm{~ms}$. What is more, this lateralized pattern of brain activity was independent of the hand being stimulated, and it is therefore suggestive of a hemispheric functional asymmetry. Third, the pattern of effects at the late time window revealing posture effects in SEPs (200 to $350 \mathrm{~ms}$ ) was also stronger at the left scalp but extended toward the central and right locations at longer latencies. Finally, these electrophysiological differences showed some correlation with the behavioural index of remapping. In particular, the magnitude of the N80 negative shift at left fronto-parietal electrodes and, to some extent, at later stage windows, was correlated with the magnitude of the crossed-hands effect in precision. These correlations, albeit weak (except for the strong correlation in the N80 time window for the left hand group), would suggests that the electrophysiological difference observed reflects neural correlates underlying tactile remapping, and more precisely the conflict between reference frames, so that bigger negative shifts index less efficient remapping. However, the inconsistent pattern in the $\mathrm{RH}$ group prevents pinpointing any solid conclusion on this result.

\section{Timing of remapping within the somatosensory network}

According to previous literature, somatosensory evoked activity starts in the human contralateral cortex by $20 \mathrm{~ms}$ after stimulation (Allison et al., 1989a, 1991), and is followed by short-latency deflections (P35, P50) thought to reflect activity confined to SI (Allison et al., 1989b; Hamalainen et al., 1990; Allison et al., 1992). From our data, it is clear that posture does 
not modulate these early processing stages. After this initial processing, activity spreads quickly throughout a network encompassing SII, posterior parietal regions (PPC) and frontal areas (e.g. premotor cortex; Mauguière et al., 1997). In particular, evidence for initial SII activity has been reported by the same latency window as our posture effects (around 70-80 ms; Mauguière et al., 1997; Forss and Jousmaki, 1998; Frot and Mauguiere, 1999; Simoes and Hari, 1999) ${ }^{4}$. This activity is very closely followed by (or contemporary to) PPC activity, according to several reports (Mauguière et al., 1997), and then by activity in frontal regions (10 ms after). Furthermore, this unfolding of neural events encompassing anterior and posterior parietal cortex as well as frontal areas is not strictly serial, since the whole network is active simultaneously for some time after stimulation (within $~ 70-150$ ms; Hari et al., 1984; Mauguière et al., 1997; Frot and Mauguiere, 1999; Schubert et al., 2008). We suggest that the early negative shift observed in the SEP reflects modulations in the initial stages of this second step of somatosensory processing, which might be driven by back-projections from the PPC to somatosensory cortices (Macaluso et al., 2000; Kitazawa, 2002; Schubert et al., 2008; see also Cauller and Kulics, 1991; Jackson and Cauller, 1998 for support from animal models and Buchholz et al. 2011, for synchronization of cortical oscillatory activity both in sensory and higher order areas during tactile remapping). Thus, the differences observed at this stage might reflect the conflict between reference frames that emerges once the external location of the touch is computed.

Based on current knowledge about the N80, one could argue that the anatomical source of the postural modulation might be SII (Mauguière et al., 1997; Forss and Jousmaki, 1998). Indeed, SII responds to postural changes (Fitzgerald et al., 2004) and some authors have suggested a role of this area in the construction of a unified body schema (Hari et al., 1993; Hari et al., 1998; Hari and Forss, 1999; Simoes and Hari, 1999). Moreover, the SIl cortex is also active both for tactile perception and action (Dijkerman and de Haan, 2007) and it seems to modulate somatosensory afferences during motor activity of the corresponding body part (Huttunen et al., 1996; Forss and Jousmaki, 1998). In line with this, some authors have hypothesised that modulations of spatial attention by changes in posture (albeit around $100 \mathrm{~ms}$ to $140 \mathrm{~ms}$ ) might arise from SII (Eimer et al., 2004; Heed and Röder, 2010). However, the role of SII in the postural modulation of touch seen here is not conclusive, first because of the lack of evidence about spatial transformations being computed in this area, and second, because as 
discussed above, several other brain areas are active during this temporal window in somatosensory processing (Mauguière et al., 1997). In fact, some studies point to regions in the PPC as being relevant for spatial transformations and in particular for tactile remapping. Good examples of these are the recent papers by Wada et al. (2012) and Takahashi et al. (2012), who found BOLD signal changes in tactile processing as a function of body posture precisely at the (left) PPC. Lloyd et al. (2003) also found differential BOLD activity evoked by touch as a function of posture in the PPC, a region the authors identified as the human homologue of monkey ventral intraparietal area (VIP; Avillac et al., 2005). Subsequent studies have supported this idea with disruption methods using TMS (Bolognini and Maravita, 2007; Azañón et al., 2010b). Yet, pinpointing VIP as the precise locus may be premature given the limited spatial resolution of fMRI and TMS and the strong involvement of several other superior and posterior parietal areas in spatial transformations, proprioceptive encoding and high-level somatosensory processing (Sakata et al., 1973; Pouget and Sejnowski, 1997; Iwamura, 1998; Graziano et al., 2000; Pellijeff et al., 2006). In fact, parietal areas 5 and 7 have been suggested to participate in somatosensory processing around this time window (about 70 to $110 \mathrm{~ms}$, Forss et al., 1994; Mauguiere et al., 1997).

\section{The laterality of remapping}

One remarkable finding of our study was the clear left lateralization of the touch-posture interaction on the SEP, which suggests a processing asymmetry. Note that despite the right and left hands were stimulated in different groups of participants, postural effects on the SEP were invariably expressed more strongly over the left scalp. Previous electrophysiological research about the effects of attention in tactile remapping has often overlooked hemispheric asymmetries by averaging across left and right electrodes (Eimer et al., 2003; Eimer et al., 2004; Röder et al., 2008; Heed and Röder, 2010) or by considering laterality effects irrelevant (Eimer et al., 2001).

The solid cerebral asymmetry found here speaks to possible different hemispheric roles during tactile spatial remapping, and fits well with the results of two recent fMRI studies involving tactile TOJ tasks comparing crossed and uncrossed hands (Takahashi et al., 2012; Wada et al., 2012). Wada et al. measured BOLD responses whilst participants adopted hands 
crossed vs. uncrossed posture. Amongst other results, they consistently observed stronger left parietal BOLD responses (including area BA40) for crossed vs. uncrossed hands. Furthermore, this BOLD response correlated significantly with participants' performance in the TOJ task (outside the scanner) so that larger crossed hands effect was associated to larger BOLD change. In yet another recent fMRI study of crossed hands, Takahashi et al. compare brain activity between arms postures for two different tactile tasks (numerosity judgments and TOJ). For the contrast relevant here, crossed vs. uncrossed posture in the TOJ task, Takahashi et al. reported significant BOLD differences in several parietal and frontal regions, with a stronger involvement of left- than right-hemisphere areas. In particular, these included left anterior parietal area BA40 (the same are activated in Wada et al. study), as well as several left frontal regions including the left PMd.

Our interpretation of this unexpected, albeit consistent, left-lateralized effect present in our study as well as in recent fMRI studies cited above relates to the fronto-parietal brain network involved in action selection and attention (Rushworth et al., 2001a, 2001b, 2003; O'Shea et al., 2007). In particular, areas in the left dorsal premotor cortex (IPMd) as well as in the left Posterior Parietal Cortex (IPPC) have been consistently associated with selection for action of the upper limbs in different contexts using fMRI and TMS (e.g., O'Shea et al., 2007; Cavina-Pratesi et al., 2006). Furthermore, failure to maintain internal representations of the body in space arising from neuropsychological deficits have been associated to left parietal lesions (Wolpert et al., 1997).

Of particular interest in the light of the present findings is the proposed dissociation between orienting covert attention and attention to $\operatorname{limb}$ movements (i.e., motor attention, according to the authors; Rushworth et al., 2001a; 2001b). The latter type of attention is supported by a left parietal area, the Supramarginal Gyrus (BA40), possibly in coordination with left PMd areas. In particular, Rushworth et al. (2001a) showed that repetitive TMS over the left (but not the right) Supramarginal Gyrus (BA40) disrupted participant's capacity to switch from one pre-planned hand movement to a new one, regardless of which hand was being used for the action. In a converging fashion, another study by Rushworth et al. (2001b) using PET with a similar task revealed significant activity in left B40, at the anterior PPC, as well as some left prefrontal regions including BA44. Note that precisely these regions are amongst the ones 
highlighted in Wada et al. 2012 and in Takahashi et al. 2012, when comparing tactile processing with hands crossed vs. uncrossed. It is perhaps worth noting that left lateralization in the parietal cortex has also been reported in relation to somehow more abstract forms of body representation, such as structural descriptions of the body (Corradi Dell'Aqua et al., 2008) and the concept of body image (Ehrsson et al., 2005). It is as yet not clear whether the tasks used in these studies could in fact be conceptualized under the framework of motor attention, but in one way or another these types of representation could in principle be also relevant in the tactile TOJ task and therefore underlie the left lateralization in our results.

Several authors, including ourselves, have proposed before that the tactile remapping process is closely linked with the action-perception loop, and that remapping a tactile stimulus into external coordinates only makes sense in the framework of acting in space (Azañon et al., 2010a, 2010b; Hermosillo et al., 2011). Supporting this point, several behavioural studies (Azañon et al., 2010a; Gallace et al., 2008) illustrate how the behavioural effects of tactile remapping in a cueing-paradigm can be strongly reduced, if not completely eliminated, when the participants' responses do not involve spatially defined actions (i.e., abstract verbal response instead of the more typical button/pedal press). In keeping with this idea, the present findings bring together the notion of tactile remapping and the concept of motor attention (or covert action planning) supported by a left lateralized network.

\section{Processing beyond the $\mathrm{N} 80$ time window}

Previous studies have found that attention effects in the P100 and N140 components are modulated by posture (Eimer et al., 2004; Gillmeister et al., 2010; Heed and Röder, 2010; Gillmeister and Forster, 2012), a finding interpreted as a reflection of tactile remapping (Eimer et al., 2004; Heed and Röder, 2010). For instance, crossing the hands abolishes the typical amplitude gain following stimulation at the attended, vs. unattended, hand (Eimer et al., 2001; Eimer et al., 2003). These results are suggestive of the moment at which the spatial allocation of attention can be expressed in terms of external coordinates, but not necessarily about the moment at which the spatial representation of the tactile event is encoded in such coordinates. Thus, the difference between our results and previous studies regarding, for example, N140, might reflect the spatial uncertainty and attentional cueing prior to the stimulus delivery in 
previous studies, as compared to the present study. Nevertheless, it is well worth noting that the late negative shift registered in the LPDa time window for the left scalp electrodes (starting at $170 \mathrm{~ms}$ ) in our results does in fact correspond to the decline process after the N140 peak. That is, the positive-going slope following the N140 peak, which culminates in the late positive deflection, was shallower in the crossed hands condition than in the uncrossed posture. It is possible that this bears some relationship with the N140 differentials registered in the attention manipulations reported in other studies, but this must remain only as a possibility at present.

\section{$\underline{\text { Conclusions }}$}

We report direct evidence for the physiological correlates of tactile remapping, and more precisely the conflict between spatial reference frames. Previous studies have provided estimates about how long it takes to complete tactile remapping, based on TOJs (Yamamoto and Kitazawa, 2001; Shore et al., 2002), crossmodal tasks (Kennett et al., 2002; Soto-Faraco et al., 2004, Azañón and Soto-Faraco, 2008a, 2008b; Azañón et al., 2010a), and saccadic trajectories (Groh and Sparks, 1996; Overvliet et al., 2011). From the present data we estimate that the neural changes leading to this outcome begin at $70-90 \mathrm{~ms}$ after tactile onset. Before this moment, a representation of touch based on external space would not be available. This is confirmed by Azañón and Soto-Faraco (2008a), who showed an advantage for visual target discrimination $60 \mathrm{~ms}$ after cueing with a touch at the anatomically corresponding (and not externally corresponding) hand. The first electrophysiological differences observed here overlap in time with the N80 component, which might be originated in SII or PPC. Previous studies have highlighted SII as the source of N80 deflections, but both SII and the PPC are indeed active during the time window relevant for the touch-posture interaction. What is more, the left PCC has been singled out as a relevant structure for remapping in fMRI studies. It is interesting however that the two TMS studies addressing the role of PPC in tactile remapping specifically (Azañon et al., 2010; Bolognini and MAravita, 2007) have targeted only the right PPC. In the light of recent fMRI results discussed above (Takahashi et al., 2012; Wada et al., 2012) and our current findings, this would suggest that despite the left hemisphere seems to be more engaged by spatial remapping of touch, the right hemisphere still plays a relevant role. More systematic studies, including TMS disruption of the left PPC, shall shed light on this issue. 
Supporting the significance of the negative shift at N80 and later stages as a neural signature of remapping, this effect correlated with the crossed-hands behavioural effect measured online. Based on temporal constrains and previous literature, our suggestion is that this marker of remapping may reflect activity in some parietal association areas (such as SII, area 5 or area 7), though even the prefrontal cortex cannot be completely excluded. What is more, interaction between several of these areas is possibly essential for successful remapping. The strong left lateralization of the effect we have found is an intriguing result that seems well confirmed by recent fMRI findings. We note the possible link between this left-lateralization and the strongly left lateralized fronto-parietal network supporting attention for action. This provides good support for the hypothesis that the brain bases underlying tactile remapping are intimately related with the perception-action network. 


\section{Acknowledgements}

We are grateful to Marco Calabresi and Cristina Rodríguez for invaluable help with the electrophysiological recordings, Josep Marco Pallarés and Cristina Baus for advice on the analyses and Xavier Mayoral for technical assistance in this project. This research was supported by the Spanish Ministry of Science and Innovation (PSI2010-15426 and Consolider INGENIO CSD2007-00012), Comissionat per a Universitats i Recerca del DIUE-Generalitat de Catalunya (SRG2009-092), and the European Research Council (StG-2010 263145). 


\section{References}

Aglioti, S., Smania, N., \& Peru A. (1999). Frames of reference for mapping tactile stimuli in brain-damaged patients. Journal of Cognitive Neuroscience, 11, 67-79.

Allison, T., McCarthy, G., Wood, C. C., Darcey, T. M., Spencer, D. D., \& Williamson, P. D. (1989a). Human cortical potentials evoked by stimulation of the median nerve. I. cytoarchitectonic areas generating short-latency activity. Journal of Neurophysiology, 62, 694-710.

Allison, T., McCarthy G., Wood, C. C., Williamson, P. D., \& Spencer, D D. (1989b). Human cortical potentials evoked by stimulation of the median nerve. II. cytoarchitectonic areas generating long-latency activity. Journal of Neurophysiology, 62, 711-722.

Allison, T., McCarthy, G., Wood, C. C., \& Jones, S. J. (1991). Potentials evoked in human and monkey cerebral cortex by stimulation of the median nerve. A review of scalp and intracranial recordings. Brain, 114, 2465-2503.

Allison, T., McCarthy, G., \& Wood, C. C. (1992). The relationship between human long-latency somatosensory evoked potentials recorded from the cortical surface and from the scalp. Electroencephalography and Clinical Neurophysiology, 84, 301-314.

Avillac, M., Deneve, S., Olivier, E., Pouget, A., \& Duhamel, J. R. (2005). Reference frames for representing visual and tactile locations in parietal cortex. Nature Neuroscience, 8, 941-949.

Azañón, E., \& Soto-Faraco, S. (2007). Alleviating the 'crossed-hands' deficit by seeing uncrossed rubber hands. Experimental Brain Research, 182, 537-548.

Azañón, E., \& Soto-Faraco, S. (2008a). Changing reference frames during the encoding of tactile events. Current Biology, 18:1044-1049.

Azañón, E., \& Soto-Faraco, S. (2008b). Spatial remapping of tactile events: Assessing the effects of frequent posture changes. Communicative \& Integrative Biology, 1, 45-46.

Azañón E., Camacho K., \& Soto-Faraco, S. (2010a). Tactile remapping beyond space. European Journal of Neuroscience, 31, 1858-1867.

Azañón, E., Longo, M. R., Soto-Faraco, S., \& Haggard, P. (2010b). The posterior parietal cortex remaps touch into external space. Current Biology, 20, 1304-1309.

Bolognini, N., \& Maravita, A. (2007). Proprioceptive alignment of visual and somatosensory maps in the posterior parietal cortex. Current Biology, 17, 1890-1895. 
Buchholz, V. N., Jensen, O., \& Medendorp, W. P. (2011). Multiple reference frames in cortical oscillatory activity during tactile remapping for saccades. Journal of Neuroscience, 31, 16864-16871.

Cauller, L. J., \& Kulics, A. T. (1991). The neural basis of the behaviorally relevant N1 component of the somatosensory-evoked potential in SI cortex of awake monkeys: Evidence that backward cortical projections signal conscious touch sensation. Experimental Brain Research, 84, 607-619.

Cavina-Pratesi, C., Valyear, K. F., Culham, J. C., Kohler, S., Obhi, S. S., Marzi, C.A., \& Goodale, M. A. (2006). Dissociating arbitrary stimulus response mapping from movement planning during preparatory period: evidence from event-related functional magnetic resonance imaging. Journal of Neuroscience, 26, 2704-2713.

Cohen, Y. E., \& Andersen, R. A. (2004). Multimodal spatial representation in the primate parietal lobe. In Spence C., \& Driver J. (Eds), Crossmodal space and crossmodal attention. New York: Oxford UP.

Collignon, O., Charbonneau, G., Lassonde, M., Lepore, F. (2009). Early visual deprivation alters multisensory processing in peripersonal space. Neuropsychologia, 47, 3236-3243.

Corradi-Dell'Acqua, C., Hesse, M. D., Rumiati, R. I., \& Fink, G. R. (2008). Where is a nose with respect to a foot? The left posterior parietal cortex processes spatial relationships among body parts. Cerebral Cortex, 18, 2879-90.

Dambacher, M., Rolfs, M., Göllner, K., Kliegl, R., \& Jacobs, A. M. (2009). Event-related potentials reveal rapid verification of predicted visual input. PLoS One, 4:e5047.

Dijkerman, H. C., \& de Haan, E. H. (2007). Somatosensory processes subserving perception and action. Behavioral \& Brain Sciences, 30, 189-201.

Duhamel, J. R., Bremmer, F., BenHamed, S., \& Graf, W. (1997). Spatial invariance of visual receptive fields in parietal cortex neurons. Nature, 389, 845-848.

Ehrsson, H. H., Kito, T., Sadato, N., Passingham, R. E., \& Naito, E. (2005). Neural substrate of body size: Illusory feeling of shrinking of the waist. PLoS Biology, 3, e412.

Eimer, M., \& Driver, J. (2000). An event-related brain potential study of cross-modal links in spatial attention between vision and touch. Psychophysiology. 37, 697-705. 
Eimer. M., Cockburn, D., Smedley, B., \& Driver, J. (2001). Cross-modal links in endogenous spatial attention are mediated by common external locations: Evidence from event-related brain potentials. Experimental Brain Research, 139, 398-411.

Eimer, M., \& Forster, B. (2003). Modulations of early somatosensory ERP components by transient and sustained spatial attention. Experimental Brain Research, 151, 24-31.

Eimer, M., Forster, B., \& Van Velzen, J. (2003). Anterior and posterior attentional control systems use different spatial reference frames: ERP evidence from covert tactile-spatial orienting. Psychophysiology, 40, 924-933.

Eimer, M., Forster, B., Fieger, A., \& Harbich, S. (2004). Effects of hand posture on preparatory control processes and sensory modulations in tactile-spatial attention. Clinical Neurophysiology, 15, 596-608.

Fitzgerald, P. J., Lane, J. W., Thakur, P. H., \& Hsiao, S. S. (2004). Receptive field properties of the macaque second somatosensory cortex: Evidence for multiple functional representations. Journal of Neuroscience, 24, 11193-11204.

Forss, N., Hari, R., Salmelin, R., Ahonen, A., Hamalainen, M., Kajola, M., Knuutila, J., \& Simola, J. (1994). Activation of the human posterior parietal cortex by median nerve stimulation. Experimental Brain Research, 99, 309-315.

Forss, N., \& Jousmaki, V. (1998). Sensorimotor integration in human primary and secondary somatosensory cortices. Brain Research, 781:259-267.

Frot, M., \& Mauguiere, F. (1999). Timing and spatial distribution of somatosensory responses recorded in the upper bank of the sylvian fissure SII area in humans. Cerebral Cortex, 9, 854-863.

Gallace, A., Soto-Faraco, S., Dalton, P., Kreukniet, B., \& Spence, C. (2008). Response requirements modulate tactile spatial congruency effects. Experimental Brain Research, 191, 171-86.

Gillmeister, H., Adler, J., \& Forster, B. (2010). Object-guided spatial attention in touch: Holding the same object with both hands delays attentional selection. Journal of Cognitive Neuroscience, 22, 931-942.

Gillmeister, H., \& Forster, B. (2012). Hands behind your back: Effects of arm posture on tactile attention in the space behind the body. Experimental Brain Research, 216, 489-497. 
Graziano, M. S., Cooke, D. F., \& Taylor, C. S. (2000). Coding the location of the arm by sight. Science, 290, 1782-1786.

Groh, J. M., \& Sparks, D. L. (1996). Saccades to somatosensory targets. I. behavioral characteristics. Journal of Neurophysiology, 75, 412-427.

Hamalainen, H., Kekoni, J., Sams, M., Reinikainen, K., \& Naatanen, R. (1990). Human somatosensory evoked potentials to mechanical pulses and vibration: Contributions of SI and SII somatosensory cortices to P50 and P100 components. Electroencephalography and Clinical Neurophysiology, 75, 13-21.

Hari, R., Reinikainen, K., Kaukoranta, E., Hamalainen, M., Ilmoniemi, R., Penttinen, A., Salminen, J., \& Teszner, D. (1984). Somatosensory evoked cerebral magnetic fields from SI and SII in man. Electroencephalography and Clinical Neurophysiology. 57, 254-263.

Hari, R., Karhu, J., Hamalainen, M., Knuutila, J., Salonen, O., Sams, M., \& Vilkman, V. (1993). Functional organization of the human first and second somatosensory cortices: A neuromagnetic study. European Journal of Neuroscience. 5, 724-734.

Hari, R., \& Forss, N. (1999). Magnetoencephalography in the study of human somatosensory cortical processing. Philosophical Transactions of the Royal Society B: Biological Sciences, 354, 1145-1154.

Hari, R., Hanninen, R., Makinen, T., Jousmaki, V., Forss, N., Seppa, M., \& Salonen, O. (1998). Three hands: Fragmentation of human bodily awareness. Neuroscience Letters, 240, 131134.

Heed, T., \& Röder, B. (2010). Common anatomical and external coding for hands and feet in tactile attention: Evidence from event-related potentials. Journal of Cognitive Neuroscience. 22, 184-202.

Heed, T., Backhaus, J., \& Röder, B. (2012). Integration of hand and finger location in external spatial coordinates for tactile localization. Journal of Experimental Psychology: Human Perception and Performance, 38, 386-401.

Hermosillo, R., Ritterband-Rosenbaum, A., \& van Donkelaar, P. (2011). Predicting future sensorimotor states influences current temporal decision making. Journal of Neuroscience, 31, 10019-22. 
Huttunen, J., Wikstrom, H., Korvenoja, A., Seppalainen, A. M., Aronen, H., \& Ilmoniemi, R. J. (1996). Significance of the second somatosensory cortex in sensorimotor integration: Enhancement of sensory responses during finger movements. Neuroreport, 7, 1009-1012. Iwamura, Y. (1998). Hierarchical somatosensory processing. Current Opinion in Neurobiology, 8, 522-528.

Jackson, M. E. \& Cauller, L. J. (1998). Neural activity in SII modifies sensory evoked potentials in SI in awake rats. Neuroreport, 9, 3379-3382.

Kennett, S., Eimer, M., Spence, C., \& Driver, J. (2001). Tactile-visual links in exogenous spatial attention under different postures: Convergent evidence from psychophysics and ERPs. Journal of Cognitive Neuroscience, 13, 462-478.

Kennett, S., Spence, C., \& Driver, J. (2002). Visuo-tactile links in covert exogenous spatial attention remap across changes in unseen hand posture. Perception and Psychophysics, 64, 1083-1094.

Kitazawa, S. (2002). Where conscious sensation takes place. Consciousness and Cognition, $11,475-477$.

Kóbor, I., Furedi, L., Kovacs, G., Spence, C., \& Vidnyanszky, Z. (2006). Back-to-front: Improved tactile discrimination performance in the space you cannot see. Neuroscience Letters, 400, 163-167.

Lloyd, D. M., Shore, D. I., Spence, C., Calvert, G. A. (2003). Multisensory representation of limb position in human premotor cortex. Nature Neuroscience, 6, 17-18.

Luck, S. J. (2005). Ten simple rules for designing ERP experiments. In: Handy, T. C. (Ed) Event-related potentials. A methods Handbook. Cambridge, MA: MIT Press.

Macaluso, E., Frith, C.D., \& Driver, J. (2000). Modulation of human visual cortex by crossmodal spatial attention. Science, 289, 1206-1208.

Maris, E., \& Oostenveld, R. (2007). Nonparametric statistical testing of EEG- and MEG-data. Journal of Neuroscience Methods, 164, 177-190.

Mauguière, F., Merlet, I., Forss, N., Vanni, S., Jousmaki, V., Adeleine, P., \& Hari, R. (1997). Activation of a distributed somatosensory cortical network in the human brain. A dipole modelling study of magnetic fields evoked by median nerve stimulation. part I: Location and 
activation timing of SEF sources. Electroencephalography and Clinical Neurophysiology, $104,281-289$.

Megela, A. L. \& Teyler, T. J. (1979). Habituation and the human evoked potential. Journal of Comparative and Physiological Psychology, 93, 1154-1170

Miyazaki, M., Yamamoto, S., Uchida, S., \& Kitazawa, S. (2006). Bayesian calibration of simultaneity in tactile temporal order judgment. Nature Neuroscience, 9, 875-877.

Moscovitch, M., \& Behrmann, M. (1994). Coding of spatial information in the somatosensory system: Evidence from patients with neglect following parietal lobe damage. Journal of Cognitive Neuroscience, 6:151-155.

Nicoletti, R., Umilta, C., \& Ladavas, E. (1984). Compatibility due to the coding of the relative position of the effectors. Acta Psychologica (Amst), 57, 133-143.

O'Shea, J., Johansen-Berg, H., Trief, D., Gobel, S., \& Rushworth, M. F. (2007). Functionally specific reorganization in human premotor cortex. Neuron, 54, 479-490.

Overvliet, K. E., Azañón, E., \& Soto-Faraco, S. (2011). Somatosensory saccades reveal the timing of tactile spatial remapping. Neurophychologia, 49, 3046-3052.

Pellijeff, A., Bonilha, L., Morgan, P. S., McKenzie, K., \& Jackson, S. R. (2006). Parietal updating of limb posture: An event-related fMRI study. Neuropsychologia, 44, 2685-2690.

Pouget, A., \& Sejnowski, T. (1997). Spatial transformations in the parietal cortex using basis functions. Journal of Cognitive Neuroscience, 9, 222-237.

Röder, B., Rösler, F., \& Spence, C. (2004). Early vision impairs tactile perception in the blind. Current Biology, 14, 121-124.

Röder, B., Focker, J., Hotting, K., \& Spence, C. (2008). Spatial coordinate systems for tactile spatial attention depend on developmental vision: Evidence from event-related potentials in sighted and congenitally blind adult humans. European Journal of Neuroscience, 28, 475483.

Rosner, B. (1975). On the detection of many outliers. Technometrics, 17, 221-227.

Sakata, H., Takaoka, Y., Kawarasaki, A., \& Shibutani, H. (1973). Somatosensory properties of neurons in the superior parietal cortex area 5 of the rhesus monkey. Brain Research, 64, 85-102. 
Rushworth, M. F., Krams, M., \& Passingham, R. E. (2001a). The attentional role of the left parietal cortex: The distinct lateralization and localization of motor attention in the human brain. Journal of Cognitive Neuroscience, 13, 698-710.

Rushworth, M. F., Ellison, A., \& Walsh, V. (2001b). Complementary localization and lateralization of orienting and motor attention. Nature Neuroscience, 4, 656-661.

Rushworth, M. F., Johansen-Berg, H., Gobel, S. M., \& Devlin, J. T. (2003). The left parietal and premotor cortices: Motor attention and selection. Neuroimage, 20, S89-S100.

Schicke, T., \& Röder, B. (2006). Spatial remapping of touch: Confusion of perceived stimulus order across hand and foot. Proceedings of the National Academy of Sciences, 103, 1180811813.

Schlack, A., Sterbing-D'Angelo, S. J., Hartung, K., Hoffmann, K. P., \& Bremmer, F. (2005). Multisensory space representations in the macaque ventral intraparietal area. Journal of Neuroscience, 25, 4616-4625.

Schubert, R., Ritter, P., Wustenberg, T., Preuschhof, C., Curio, G., Sommer, W., \& Villringer, A. (2008). Spatial attention related SEP amplitude modulations covary with BOLD signal in S1a simultaneous EEG- fMRI study. Cerebral Cortex, 18, 2686-2700.

Shore, D. I., Spry, E., \& Spence, C. (2002). Confusing the mind by crossing the hands. Cognitive Brain Research, 14, 153-163.

Simoes, C. \& Hari, R. (1999). Relationship between responses to contra- and ipsilateral stimuli in the human second somatosensory cortex SII. Neuroimage, 10, 408-416.

Soto-Faraco, S., Ronald, A., \& Spence, C. (2004). Tactile selective attention and body posture: Assessing the multisensory contributions of vision and proprioception. Perception and Psychophysics, 66, 1077-1094.

Spence, C., Pavani, F., \& Driver, J. (2004). Spatial constraints on visual-tactile cross-modal distractor congruency effects. Cognitive, Affective, \& Behaviorial Neuroscience, 4, 148-169.

Takahashi, T., Kansaku, K., Wada, M., Shibuya, S., \& Kitazawa, S. (in press). Neural correlates of tactile temporal-order judgment in humans: An fMRI study. Cerebral Cortex.

Thorpe, S., Fize, D., \& Marlot, C. (1996). Speed of processing in the human visual system. Nature, 381, 520-522. 
Tomberg, C., Desmedt, J. E., Ozaki, I., Nguyen, T. H., \& Chalklin, V. (1989). Mapping somatosensory evoked potentials to finger stimulation at intervals of 450 to $4000 \mathrm{msec}$ and the issue of habituation when assessing early cognitive components. Electroencephalography and Clinical Neurophysiology/Evoked Potentials Section, 74, 347358.

Wada, M., Yamamoto, S., \& Kitazawa, S. (2004). Effects of handedness on tactile temporal order judgment. Neuropsychologia, 42, 1887-1895.

Wada, M., Takano, K., Ikegami, S., Ora, H., Spence, C., \& Kansaku, K. (2012). Spatio-temporal updating in the left posterior parietal cortex. PLoS One, 7, e39800.

Wolpert, D. M., Goodbody, S. J., Husain, M. (1998). Maintaining internal representations: The role of the human superior parietal lobe. Nature Neuroscience, 1, 529-533.

Yamamoto, S. \& Kitazawa, S. (2001). Reversal of subjective temporal order due to arm crossing. Nature Neuroscience, 4, 759-765.

Yamamoto, S., Miyazaki, M., Iwano, T., \& Kitazawa, S. (2012). Bayesian calibration of simultaneity in audiovisual temporal order judgments. PLoS One, 7, e40379.

Yates, M. J., \& Nicholls, M. E. R. (2009). Somatosensory prior entry. Perception \& Psychophysics, 71, 847-859.

Yates, M. J., \& Nicholls, M. E. R. (2011). Somatosensory prior entry assessed with temporal order judgments and simultaneity judgments. Attention, Perception \& Psychophysics, 73, $1586-1603$ 


\section{Footnotes:}

1. For this test, which deals with the multiple comparison problem, we selected a temporal window of $40 \mathrm{~ms}$ around the time window that survived the significance threshold for each electrode and calculated a paired t-test value (i.e., crossed vs. uncrossed) for each time point. We then clustered the data that resulted larger than the threshold of $P<.05$ and followed the temporal adjacency criteria of at least $10 \mathrm{~ms}$, computed the sum of the squares of the $t$ values and chose the maximum cluster level statistic. The permutation distribution was then obtained by (1) randomly re-assigning each data point in the two conditions (i.e. crossed vs uncrossed) to two equivalent data sets at random; (2) for each cluster, we computed again the squared sum of t values and then applying a comparison test statistic equal to the maximum of the cluster level statistic, and (3) repeated steps one and two 2000 times to construct an histogram. The nonparametric statistical test was finally estimated by calculating a "Monte Carlo" p value under the permutation distribution and comparing it with an alpha level of 0.05 .

2. Note that the amplitude of these somatosensory evoked potentials is relatively small compared to some other studies (Eimer and Driver, 2000; Eimer et al., 2001; Eimer et al., 2003; Gillmeister et al., 2010). One reason is that we used a weak tactile stimulus (brief mechanical tap of $9 \mathrm{~ms}$ ), instead of electrical stimulation of the medial nerve of ERP/MEG studies or the considerably longer vibrotactile stimuli (of about $200 \mathrm{~ms}$ ) used in some other ERP studies. In our case, this type of stimulation was used to bring performance within measurable range in the behavioural task and to prevent the temporal overlap between stimulation and evoked potential. Another additional reason for the small SEPs is habituation (Megela, and Teyler, 1979; Tomberg et al., 1989) since our stimulation was repeatedly presented at one single finger (to prevent fluctuation of attention in single tap trials; $90 \%$ of the trials).

3. To explore this idea further, we confirmed the previous results by submitting the data to a new set of analyses including data from all the participants that were excluded from analyses due to poor performance in the behavioural test ( $\mathrm{LH}$ group: 4; $\mathrm{RH}$ group 4). The addition of these new data $(n=40)$ strengthened some of the interactions seen in the main analysis. Again, 
for the N80 time window a Posture $x$ Hemisphere interaction was found $\left.\left[F_{(1,38)}=4.8 ; P=.035\right)\right]$. The three way interaction, posture $\mathrm{x}$ hemisphere $\mathrm{x}$ coronal position, marginal in the previous analyses, was now significant $\left[F_{(1,38)}=6.9, P=.01\right]$. The ANOVA for the LPDa returned again a main effect of Posture $\left[F_{(1,38)}=6.6, P=.014\right]$, a more clear Posture $\times$ Hemisphere interaction $\left[F_{(1,38}=8.8, P=.005\right]$, and Posture $\times$ Coronal $\times$ Sagittal per group interaction $\left[F_{(1.9,75.8)}=3.7, P=.03\right]$. The Posture $\mathrm{x}$ hemisphere $\mathrm{x}$ Sagittal $\mathrm{x}$ group interaction $\left[F_{(2.2,84)}=4.8, P=.008\right]$ was the only new effect with respect to this window. Finally, in the LPDb window, we observed again the main effect of Posture $\left[F_{(1,38)}=7.2, P=.011\right]$, as well as the two interactions previously detected; an increased Posture $\mathrm{x}$ Hemisphere interaction $\left[F_{(1,38}=8.9, P=.005\right]$, an a Posture $\mathrm{x}$ Coronal position $\left[F_{(1,38)}=4.5, P=.04\right]$.

4. Note that some authors have reported SII activity as early as $60 \mathrm{~ms}$, but at variance with our paradigm using mechanical stimulation at the finger, they have used electrical stimulation of the medial nerve (Frot and Mauguiere, 1999). 


\section{Figure Legends}

Equation 1. Four-parameter cumulative Gaussian function used to fit the behavioural data. $t$ is the time interval between left and right tap negative is left first, $P_{\max }$ is the upper bound, $P_{\min }$ is the lower bound, $d$ is the mean of the function and, $\sigma$ is the standard deviation. The mean of the function corresponds to the point of subjective simultaneity PSS and the standard deviation corresponds to the temporal resolution or Just Noticeable Difference at an $84 \%$ threshold.

Figure 1. Behavioural results of the left hand study (top) and right hand study (bottom). The left plot of each panel represents the proportion of right hand first responses as a function of the stimulus onset asynchrony SOA (negative represents left hand first). Crossed posture is represented in black and uncrossed in grey. The right plot of each panel represents the probability of judgment reversals in the crossed posture with respect to the uncrossed posture, where positive denotes right-to.left-hand reversal (vice-versa for negative) across SOAs (same convention as before).

Figure 2. Grand-average event-related potentials and topographical maps. A. Grandaverage somatosensory-evoked potential for left (top) and right hand stimulation (bottom) at one ipsi- and one contralateral electrode (FT7 and C4). Blue and red lines represent crossed- and uncrossed-hand postures, respectively. The main somatosensory components visible in the signal are highlighted in one of the graphs. B. The topographic maps show the average voltage difference between crossed and uncrossed posture (crossed-uncrossed subtraction; Left hand study, top; Right hand study, bottom) for each of the temporal windows used for analysis (see text). Blue is negative, red is positive. Electrodes that yielded significant differences across postures are marked in yellow $(P<.05)$.

Figure 3. Time by electrode significance maps for the comparison between SEPs corresponding to crossed and uncrossed postures. Left hand stimulation group is presented in left panel, and the right hand stimulation group in the right panel. Color represents p-values obtained after the computation of paired t-tests between crossed and uncrossed 
conditions at each electrode and time point (see color key). The sections underlined in black correspond to clusters of consecutive points with $\mathrm{t}$-values with $P<.05$ significance level that survived the M/EEG-cluster-based nonparametric permutation test (Maris and Oostenveld, 2007). Electrodes are grouped by laterality from top to bottom (left, central and right), and by proximity. The first reliable significant difference is circled with a white dotted line.

Figure 4. Correlations between behavioral performance and the size of the negative shift in ERPs within the N80 window (left hand group only). Electrode by electrode correlation between the crossed hands deficit (difference in precision between crossed and uncrossed hands) and the size of the negative shift in SEPs within the N80 window. Within each graph, each dot corresponds to one participant. Yellow dots and lines represents significant correlation (all r2>.46, Bonferroni correction for multiple comparisons, $P<.0028$ ). 
EQUATION1.

$$
P(t)=\left(P_{\max }-P_{\min }\right) \int_{-\infty}^{t} \frac{1}{\sqrt{2 \pi \sigma_{u}}} e^{\frac{-\left(\tau-d_{u}\right)^{2}}{2 \sigma_{u}^{2}}} d \tau+P_{\min }
$$




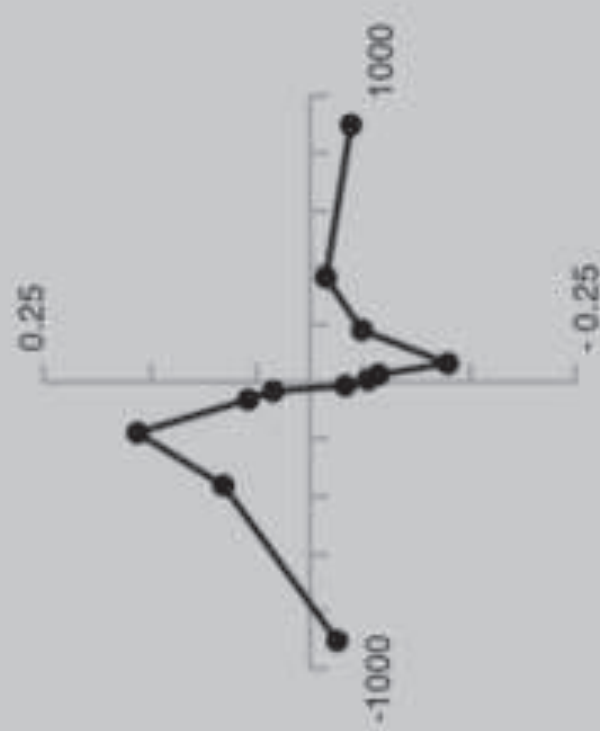

sjessenas dosd

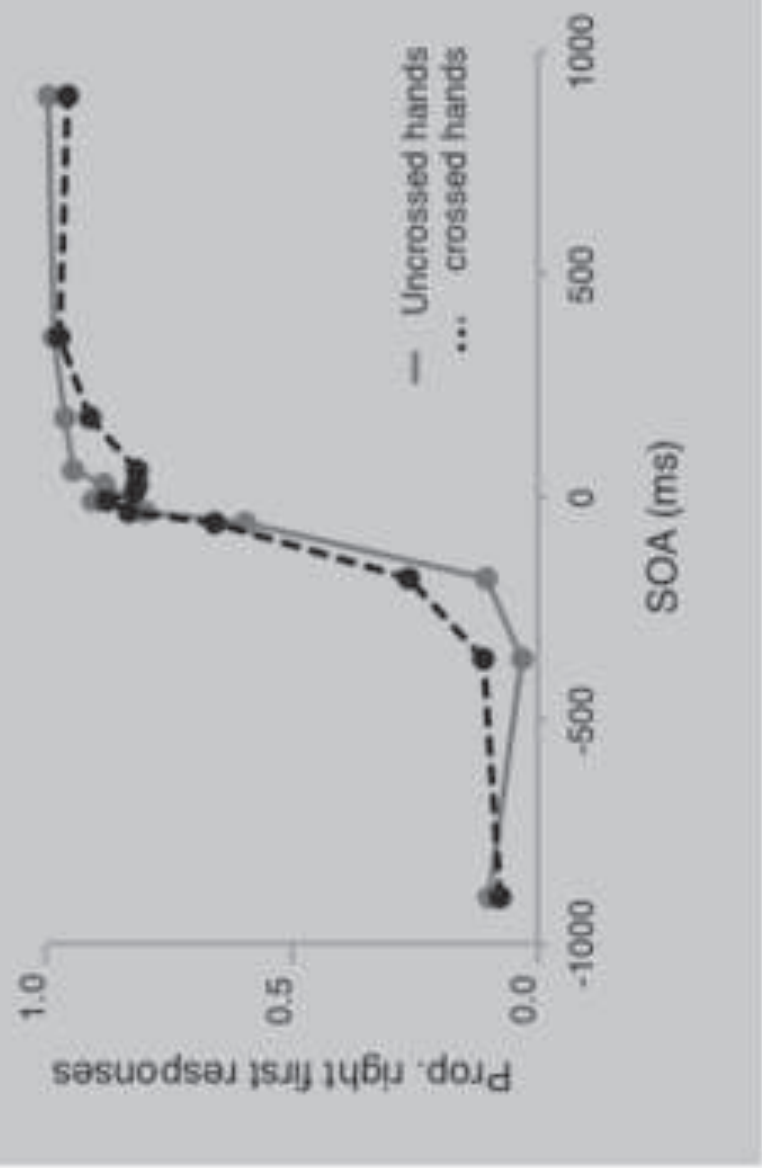

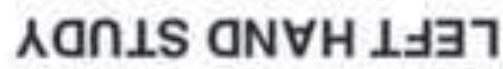

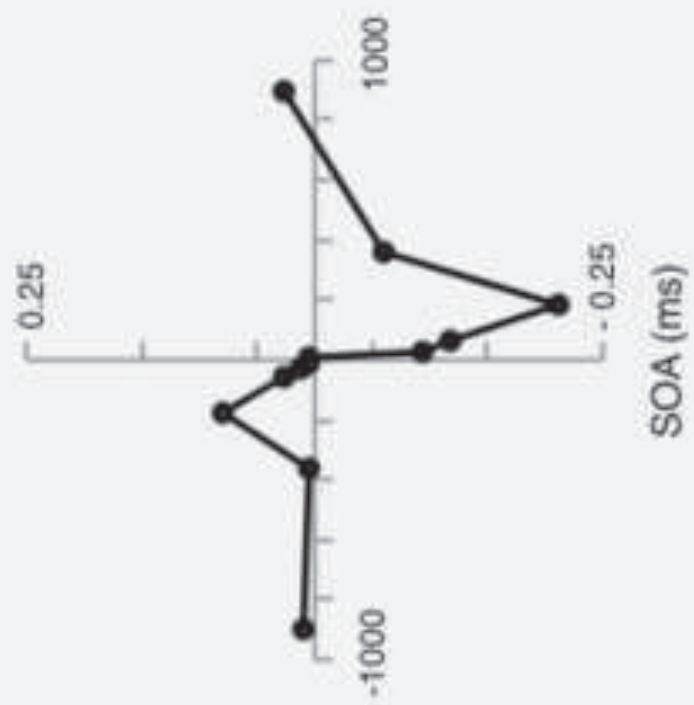

sjesjanas doud

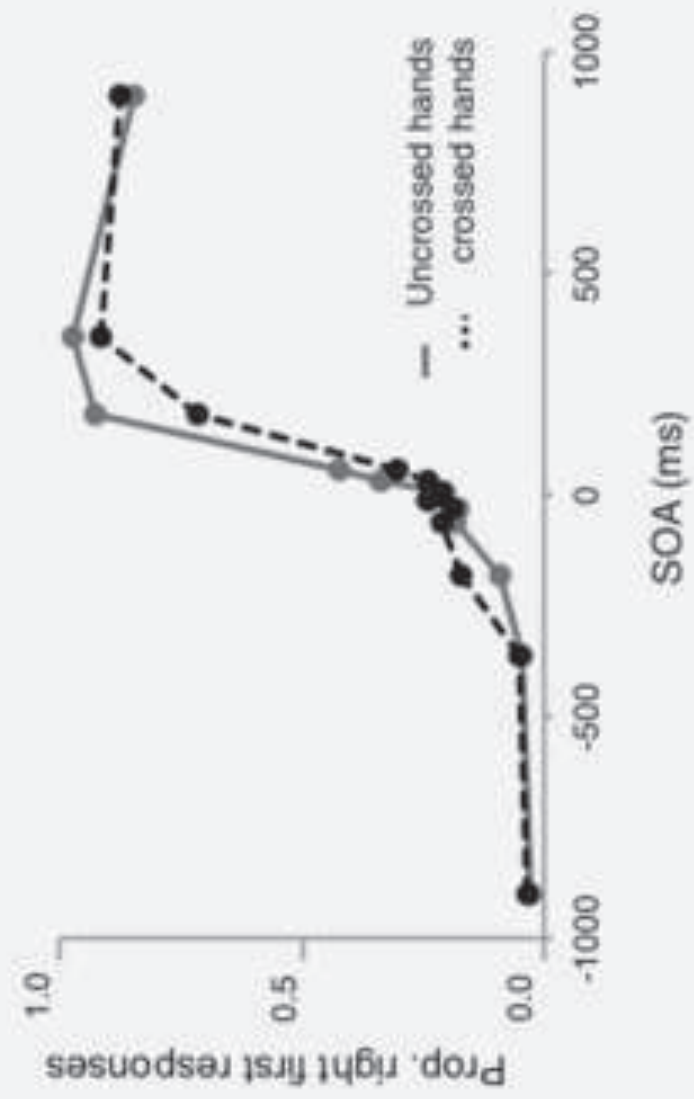

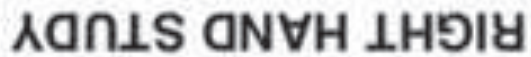


A
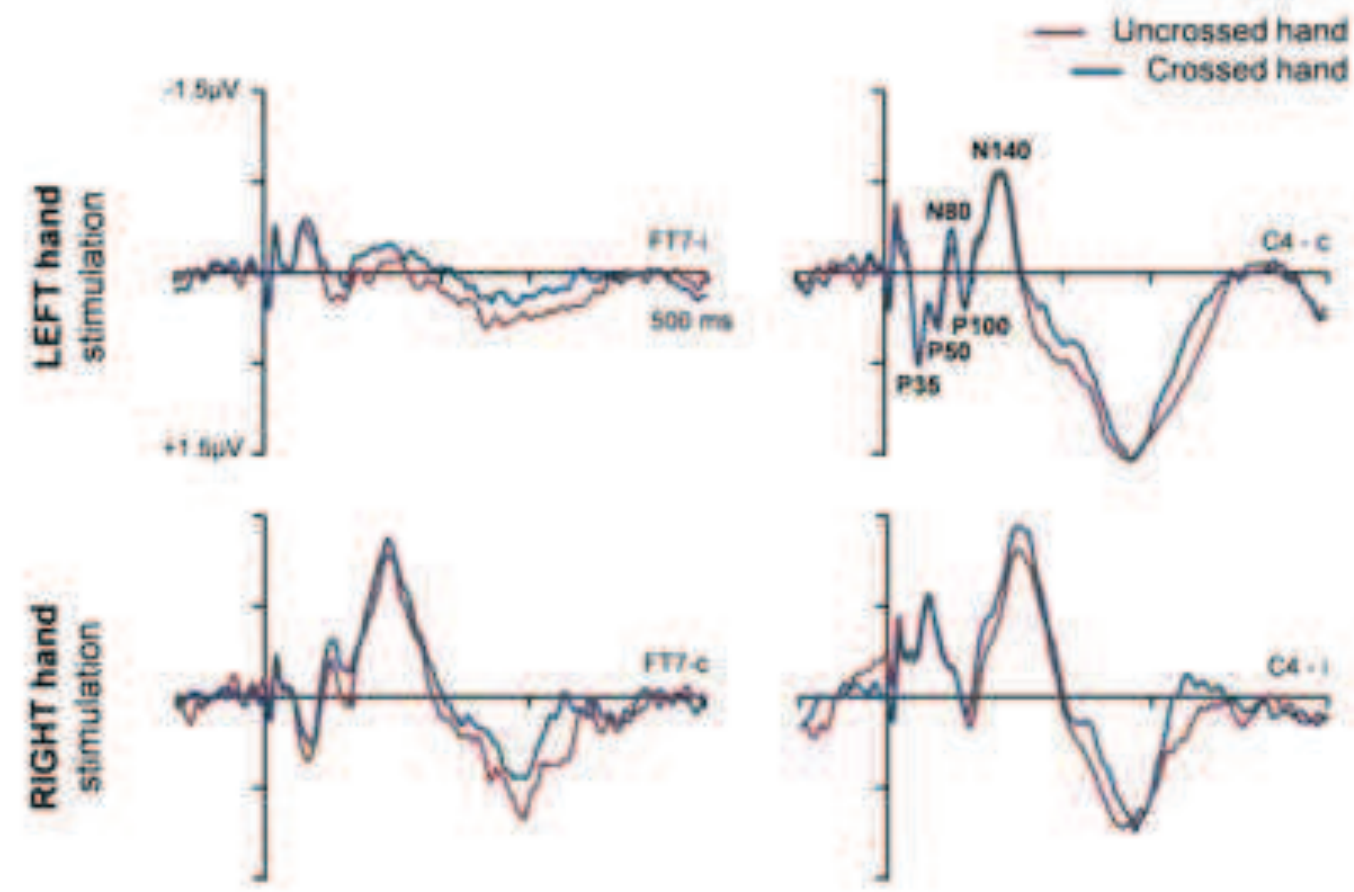

B
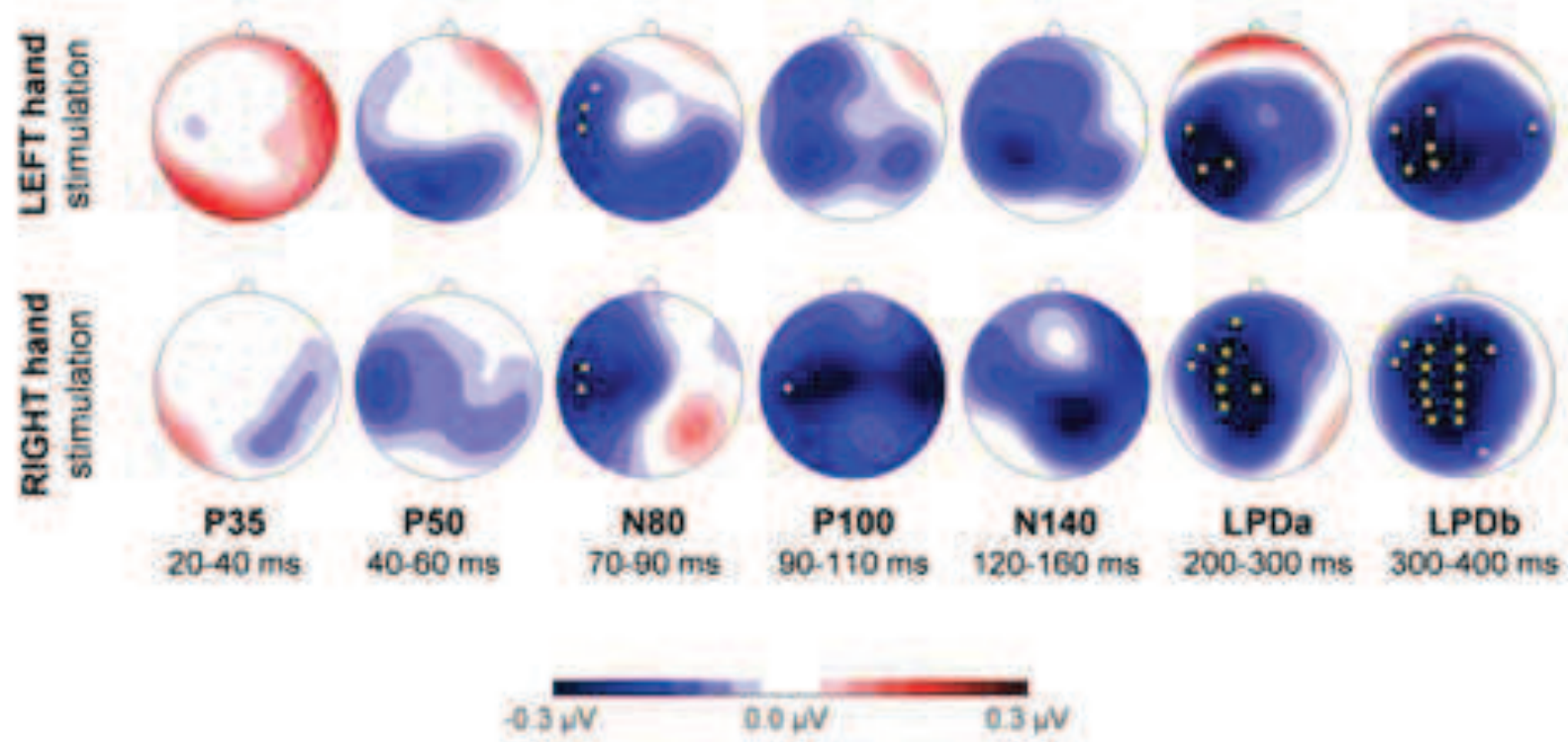


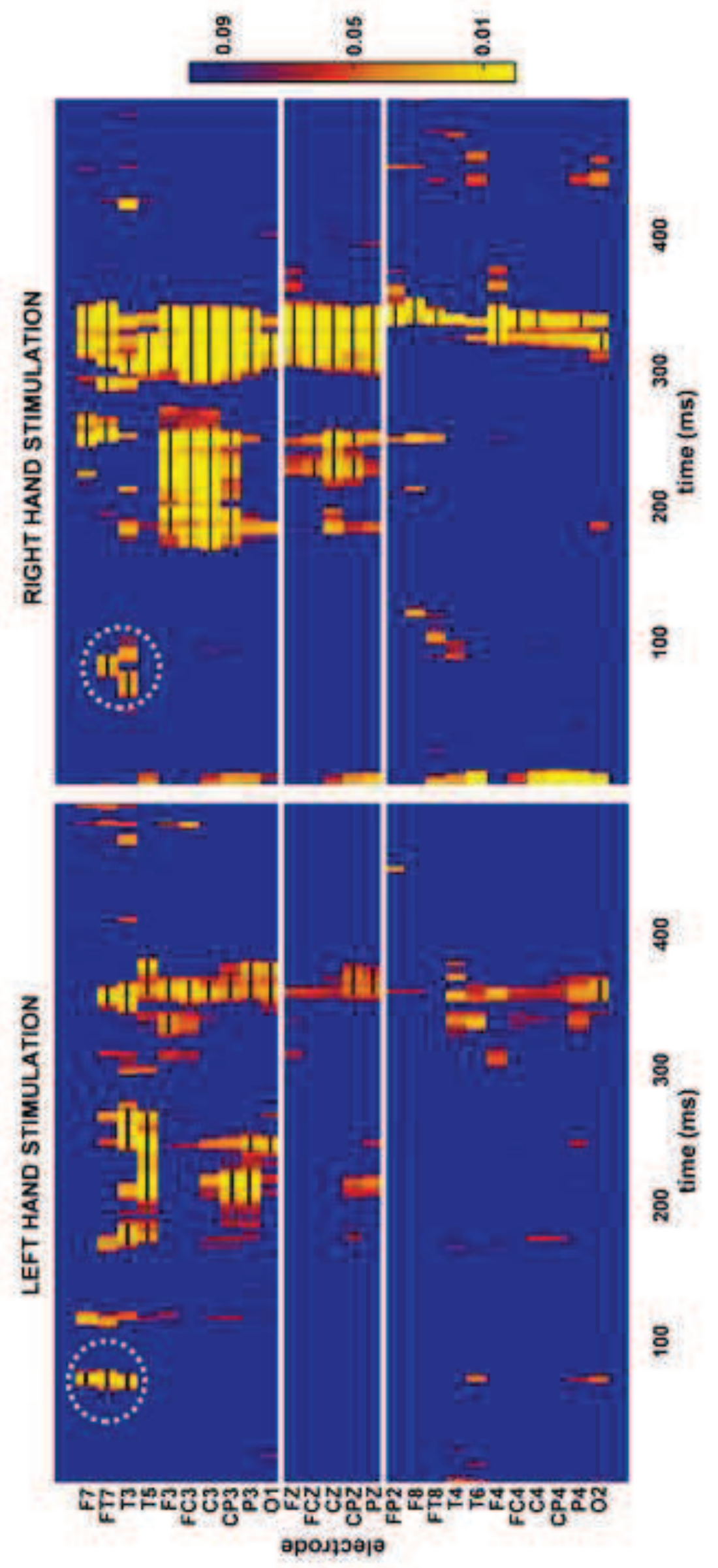

0

次

은 


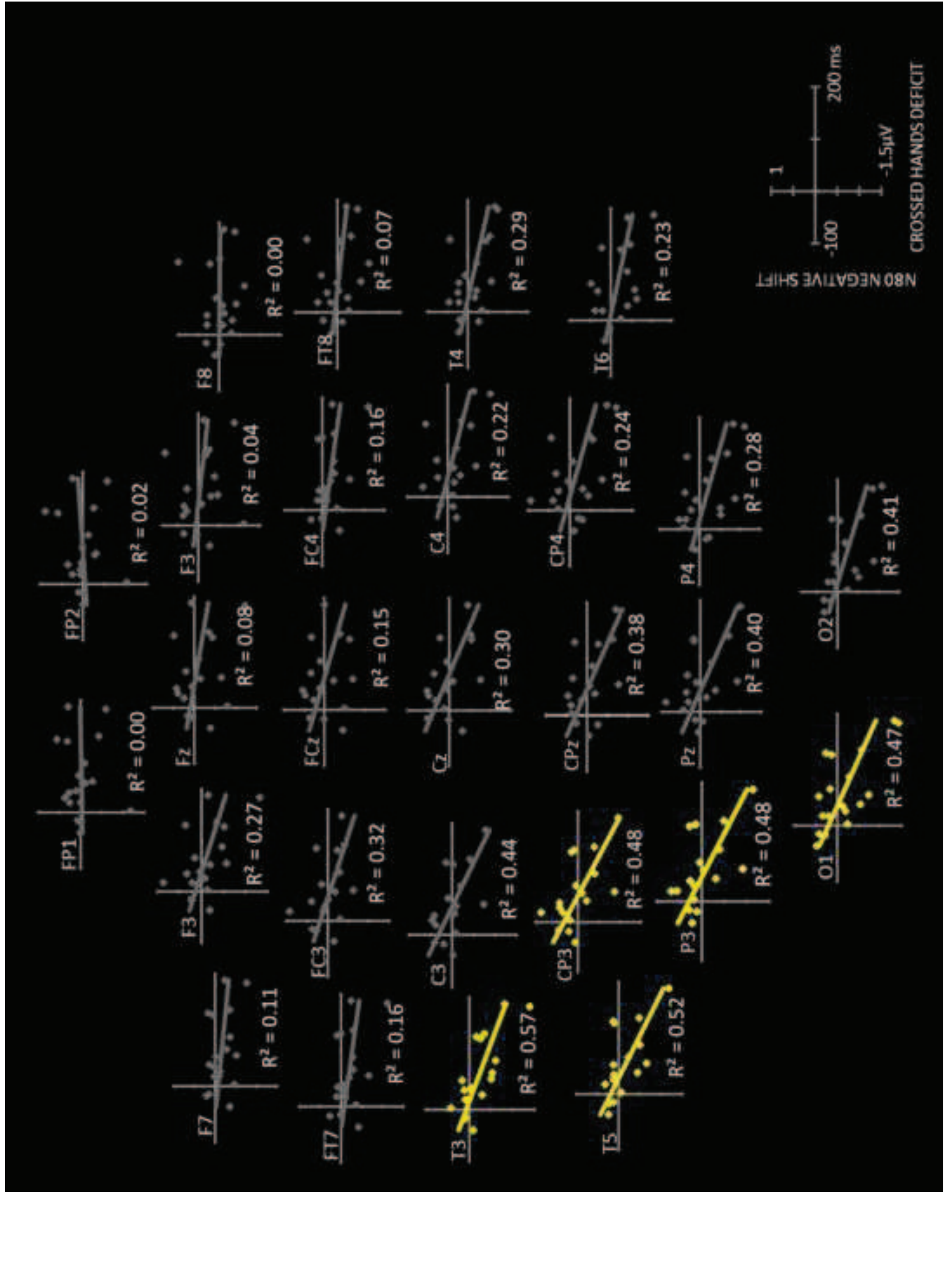

